\title{
Bridge condition assessment from long-term monitoring by means of Bayesian hypothesis test
}

\section{$\operatorname{AUTHOR}(S)$ :}

Kim, C. W.; Wang, Z.; Morita, T.; Kawatani, M.; Takase, $\mathrm{K}$.

\section{CITATION:}

Kim, C. W. ... [et al]. Bridge condition assessment from long-term monitoring by means of Bayesian hypothesis test. Life-Cycle of Engineering Systems: Emphasis on Sustainable Civil Infrastructure 2016: 713-720

ISSUE DATE:

2016-10-04

URL:

http://hdl.handle.net/2433/255547

\section{RIGHT:}

This is an Accepted Manuscript of a book chapter published by Routledge/CRC Press in Life-Cycle of Engineering Systems: Emphasis on Sustainable Civil Infrastructure on 4 October 2016, available online:

http://www.routledge.com/9781138028470; この論文は出版社版でありません。引用の際には出版社版をご確認ご利 用ください。; This is not the published version. Please cite only the published version. 


\title{
Bridge condition assessment from long-term monitoring by means of Bayesian hypothesis test
}

\author{
C.W. Kim and Z. Wang \\ Dept. of Civil and Earth Resources Eng., Kyoto University, Kyoto 615-8540, Japan \\ T. Morita \\ Obayashi Corporation, Japan \\ M. Kawatani \\ Dept. of Civil Eng., Kobe University, Kobe 657-8501, Japan \\ K. Takase \\ OMRON Social Solutions CO., LTD, Japan
}

\begin{abstract}
This study presents an approach to reduce effects of environmental and operational factors on long-term monitoring data of bridges. The Bayesian approach comprising both Bayesian regression and Bayesian hypothesis test is applied to investigate monitoring data of an in-service seven-span plate-Gerber bridge. This study considers time-varying temperature and vehicle weights as environmental and operational factors respectively. Vehicle weights were measured utilizing a bridge weigh-in-motion (BWIM) system installed on the bridge. All data was taken from a healthy bridge, since no damage and deterioration was reported during the monitoring period. Observations through the study demonstrated that considering both temperature and vehicle weight as environmental and operational factors in Bayesian regression led to improved regression results than that considering only temperature. It also showed that monitoring the data observed at a specific time could reduce influence of traffic in long-term monitoring. In the Bayesian hypothesis testing utilizing data from the healthy bridge, the bridge was judged as healthy.
\end{abstract}

\section{INTRODUCTION}

Maintaining and improving civil infrastructures including bridge structures are keen technical issues in many countries. Developing an effective maintenance strategy relies on a timely decision on the health condition of the structure. Structural health monitoring (SHM) using vibration data thus has been recognized as one of the promising technologies for providing a timely decision on the bridge health condition. Most precedent studies on SHM specifically examine changes in modal properties of structures (e.g. Doebling et al. 1996). The fundamental concept of this technology is that modal parameters are functions of structures' physical properties. Therefore, changes in physical properties, such as reduced stiffness resulting from damage, will detectably change these modal properties.

In fact, many techniques to identify the hidden information of structural integrity in the vibration data have been proposed to diagnose bridge structures (Deraemaeker et al. 2007, Dilena and Morassi 2011 and Kim et al. 2012). However, real bridge structures are subject to changing environmental and operational conditions that affect structural integrity information during the long in- service period. Some components in the signals affected by those environmental and operational effects lurk in the measured vibration data and disguise themselves as structural responses (Sohn et al. 2003, and Deraemaeker et al. 2007).

As in-service effects on vibration monitoring of bridges, temperature, wind and traffic-induced vibrations and traffic mass effects are factors to be considered. Focusing on short and medium span bridges, however, temperature and traffics are dominant factors affecting the vibration of bridges (Peeters and De Roeck 2001, and Cunha et al. 2013). Therefore, how to consider those environmental and operational effects is a crucial issue in long-term bridge health monitoring.

This study is intended to investigate a way to consider time-varying temperature and vehicle weight as environmental and operational factors for long-term vibration-based bridge health monitoring (BHM) by means of a Bayesian approach, which is an improvement from previous researches considering only temperature as an environmental factor by Kim et al. (2011 and 2013a).

The proposed Bayesian approach consists of three steps. Step 1 is to identify damage indicators (DIs) from coefficients of the auto-regressive (AR) model utilizing bridge accelerations (Nair et al. 2006, Kim 
et al. 2012 and 2013b). Since AR coefficients are closely linked with features of bridge vibrations, changes in DI are not only caused by changes in the bridge health condition but also by changes of environmental and operational conditions during monitoring. Step 2 is to perform a regression analysis of the DIs identified in Step 1 (observed DI $\left.\left(\mathrm{DI}_{o b}\right)\right)$ to consider environmental and operational changes by means of the Bayesian regression (Kitagawa and Gersch 1984) to examine long-term monitoring data effectively by an online updating. Step 3 is the step for decision making on the bridge health condition by means of the Bayesian hypothesis test (Sankararaman and Mahadevan 2011) in which residuals estimated from differences between the $\mathrm{DI}_{o b}$ and the predicted DI (DI $\left.p r\right)$ estimated by the Bayesian regression in Step 2.

The Bayesian approach is applied to investigate monitoring data of an in-service seven-span plateGerber bridge. This study considers time-varying temperature and vehicle weight as environmental and operational factors respectively. Vehicle weights are identified utilizing a bridge weigh-inmotion (BWIM) system (Moses 1979 and Heng et al. 2011) installed on the bridge. All the data is taken from the healthy bridge, since no damage and deterioration was reported during the monitoring period. The influences of those environmental and operational factors on regression are also investigated by comparing the following three cases: consider both temperature and vehicle weight, consider only temperature, and consider only vehicle weight.

\section{A BAYESIAN APPROACH FOR LONG-TERM BHM}

\subsection{Identification of damage-sensitive features from AR coefficients: Step 1}

Many studies focus on changes in system frequencies and structural damping constants for the structural diagnosis of bridges utilizing a linear time series model such as the AR model. However, there exist drawbacks in modal parameter-based bridge diagnosis using time series models; e.g., the optimal time series model for vibration responses of bridge structures usually comprises a higher-order term, and as a result the optimal model identifies even spurious modal parameters, which causes false system frequencies and damping constants. Those false modal parameters make it difficult to choose the proper modal parameters affected by structural damage. The drawback of the existing method is the driving force behind this study. This study considered an alternative parameter based on AR coefficients as a damage-sensitive feature for the vibration-based BHM because both system frequency and damping constant are related to $\mathrm{AR}$ coefficients (Nair et al. 2006).

Step 1 is to identify the $\mathrm{DI}_{o b}$ from coefficients of the AR model of bridge acceleration responses. This paper includes only a brief description about the DI without covering details, since theoretical backgrounds of utilizing AR coefficients as a damage-sensitive feature have already investigated by Nair et al. (2006) for a model building structure and by Kim et al. (2013b) for a model bridge and thus details can be fully accessed in the studies.

The linear dynamic system can be idealized using the AR model shown in Equation 1,

$z_{k}=\sum_{i=1}^{p} a_{i} z_{k-i}+e_{k}$

where $z_{k}$ denotes the $k$-th output of a system, $a_{i}$ is the $i$-th AR coefficient, $p$ is the optimal AR order and $e_{k}$ indicates the $k$-th error. The optimal AR order, which is obtainable by means of Akaike Information Criteria (AIC) (Akaike 1974), is used in this study. AIC is given by Equation 2 .

$$
\mathrm{AIC}=n \log \left(2 \pi E^{2}\right)+2(m+1)+n
$$

where $n$ indicates the number of data, $m$ represents $\mathrm{AR}$ order, and $E^{2}$ means square of prediction error. The AIC consists of two terms; the first term is a log-likelihood function and the second term is a penalty function for the number of the AR order.

The $\mathrm{DI}_{o b}$ is defined by Equation 3. (Nair et al. 2006 and Kim et al. 2013b)

$$
\mathrm{DI}_{o b}=\frac{\left|a_{1}\right|}{\sqrt{a_{1}^{2}+a_{2}^{2}+a_{3}^{2}}}
$$

where $a_{1}, a_{2}$ and $a_{3}$ indicate the first, second and third AR coefficients respectively.

Nair et al. (2006) showed that the first three AR coefficients are the most significant among all the coefficients of the AR model utilizing data from the laboratory experiment on a model building. Kim et al. (2013b) also observed that the $\mathrm{DI}_{o b}$, considering up to the third order of the AR coefficients, is a promising parameter in bridge health monitoring through a bridge-moving vehicle laboratory experiment.

\subsection{Consideration of environmental and operational changes: Step 2}

In a long-term bridge monitoring, the $\mathrm{DI}_{o b}$ varies due to time-varying environmental and operational conditions. Influences of environmental and operational factors on $\mathrm{DI}_{o b}$ are treated as exterior disturbances in the ARX model. This study adopts the Kalman filter to estimate the model parameters 
with ARX model that provides relationship between DI and external disturbances caused by environmental and operational factors. The idea is to perform a regression analysis with the Kalman filter to estimate model parameters of the ARX model. The Kalman filter is adopted to realize online updating and therefore makes it possible to utilize long-term monitoring data more efficiently. The converged model parameters after updating were considered as the optimal model parameters.

\subsubsection{Kalman filter}

The state space model for the Bayesian regression is given by Equation 4 and Equation 5 .

$$
\begin{aligned}
& \mathbf{x}_{t}=\mathbf{F} \mathbf{x}_{t-1}+\mathbf{G} \mathbf{v}_{t} \\
& y_{t}=\mathbf{H}_{t} \mathbf{x}_{t}+w_{t}
\end{aligned}
$$

where $\mathbf{x}_{t}$ and $y_{t}$ are the state matrix and observation at a time $t$ respectively. $\mathbf{v}_{t}$ and $w_{t}$ denote noise at $t$. For any particular models of the time series, F, G and $\mathbf{H}_{t}$ are known. The future state can be predicted in terms of the Kalman filter as follows. (Kitagawa and Gersch 1984)

$$
\begin{aligned}
& \overline{\mathbf{x}}_{t \mid t-1}=\mathbf{F} \overline{\mathbf{x}}_{t-1 \mid t-1} \\
& \mathbf{V}_{t \mid t-1}=\mathbf{F} \mathbf{V}_{t-1 \mid t-1} \mathbf{F}^{\mathrm{T}}+\mathbf{G Q G}^{\mathrm{T}}
\end{aligned}
$$

where $\overline{\mathbf{x}}_{t \mid t-1}$ and $\mathbf{V}_{t \mid t-1}$ denote the predicted conditional mean matrix and covariance matrix at $\mathrm{t}$ under the condition of the state at $t-1$ respectively. $Q$ stands for the covariance matrix of $\mathbf{v}_{t}$. The filtered state also can be estimated as follows.

$$
\begin{aligned}
& \overline{\mathbf{x}}_{t \mid t}=\overline{\mathbf{x}}_{t \mid t-1}+\mathbf{K}_{t}\left(y_{t}-\mathbf{H}_{t} \overline{\mathbf{x}}_{t \mid t-1}\right) \\
& \mathbf{V}_{t \mid t}=\left(\mathbf{I}-\mathbf{K}_{t} \mathbf{H}_{t}\right) \mathbf{V}_{t \mid t-1}
\end{aligned}
$$

where $\mathbf{K}_{t}$ is the Kalman gain defined by Equation 10 .

$$
\mathbf{K}_{t}=\mathbf{V}_{t \mid t-1} \mathbf{H}_{t}^{\mathrm{T}}\left(\mathbf{H}_{t} \mathbf{V}_{t \mid t-1} \mathbf{H}_{t}^{\mathrm{T}}+R\right)^{-1}
$$

where $R$ denotes the variance of $w_{t}$.

\subsubsection{ARX model}

In this study, environmental and operational factors are treated as exterior disturbances in the ARX model, which can be expressed discretely as shown in Equation 11. (Kim et al. 2015)

$$
\mathrm{DI}_{o b}^{(t)}=\sum_{i=1}^{q} \alpha_{i} \mathrm{DI}_{o b}^{(t-i)}+\sum_{i=1}^{r} \beta_{i} u^{(t-i)}+w_{t}
$$

where $q$ and $r$ stand for model orders. $\alpha_{i}$ and $\beta_{i}$ are model parameters. $u^{(t)}$ denote environmental and operational factors at $t$ and $w_{t}$ is noise at $t$ respectively. In other words, this study assumes the following relationships for the parameters and matrixes in Equations 4 and 5.

$y_{t}=\mathrm{DI}_{o b}^{(t)}$

$\mathbf{F}=\mathbf{I}$ (Identity matrix)

$\mathbf{G}=\mathbf{0}$ (Null matrix)

$\mathbf{x}_{t}=\left[\alpha_{1}, \ldots, \alpha_{q}, \beta_{1}, \ldots, \beta_{r}\right]^{\mathrm{T}}$

$\mathbf{H}_{t}=\left[\mathrm{DI}_{o b}^{(t-1)}, \ldots, \mathrm{DI}_{o b}^{(t-q)}, u^{(t-1)}, \ldots, u^{(t-r)}\right]$

$R=1$

Moreover the initial mean and covariance matrixes were assumed as follows,

$\overline{\mathbf{x}}_{0 \mid 0}=\mathbf{0}$ (Null matrix)

$\mathbf{V}_{0 \mid 0}=\mathbf{I}($ Identity matrix $)$

The optimal model parameters are obtained by satisfying Equation 20.

$\left|\overline{\mathbf{x}}_{t \mid t}-\overline{\mathbf{x}}_{t-1 \mid t-1}\right| \cong 0$

\subsection{Anomaly detection utilizing the Bayesian hypothesis test: Step 3}

Step 3 is for anomaly detection by means of the Bayesian hypothesis test based on residuals estimated by Equation 21 .

$r_{t}=D I_{o b}(t)-D I_{p r}(t)$

where $D I_{p r}(t)=\mathbf{H}_{t} \mathbf{x}_{\mathrm{opt}}$.

\subsubsection{Bayes factor}

In Bayesian statistics, it is possible to estimate the probability of a hypothesis conditionally on observed data. If the null hypothesis $\left(\mathrm{H}_{0}\right)$ is defined as 'healthy' and the alternate hypothesis $\left(\mathrm{H}_{1}\right)$ is defined as 'damage', the posterior odds are obtained by utilizing priors and marginal likelihoods as follows.

$$
\text { Post }_{\text {odds }}=\frac{p\left(\mathrm{H}_{1} \mid \mathbf{D}\right)}{p\left(\mathrm{H}_{0} \mid \mathbf{D}\right)}=\frac{p\left(\mathbf{D} \mid \mathrm{H}_{1}\right)}{p\left(\mathbf{D} \mid \mathrm{H}_{0}\right)} \times \frac{p\left(\mathrm{H}_{1}\right)}{p\left(\mathrm{H}_{0}\right)}
$$

where D refers to the data obtained during monitoring, $p\left(\mathrm{H}_{1}\right) / p\left(\mathrm{H}_{0}\right)$ indicates the prior odds, $p\left(\mathbf{D} \mid \mathrm{H}_{1}\right)$ and $p\left(\mathbf{D} \mid \mathrm{H}_{0}\right)$ are called the marginal likelihoods. 


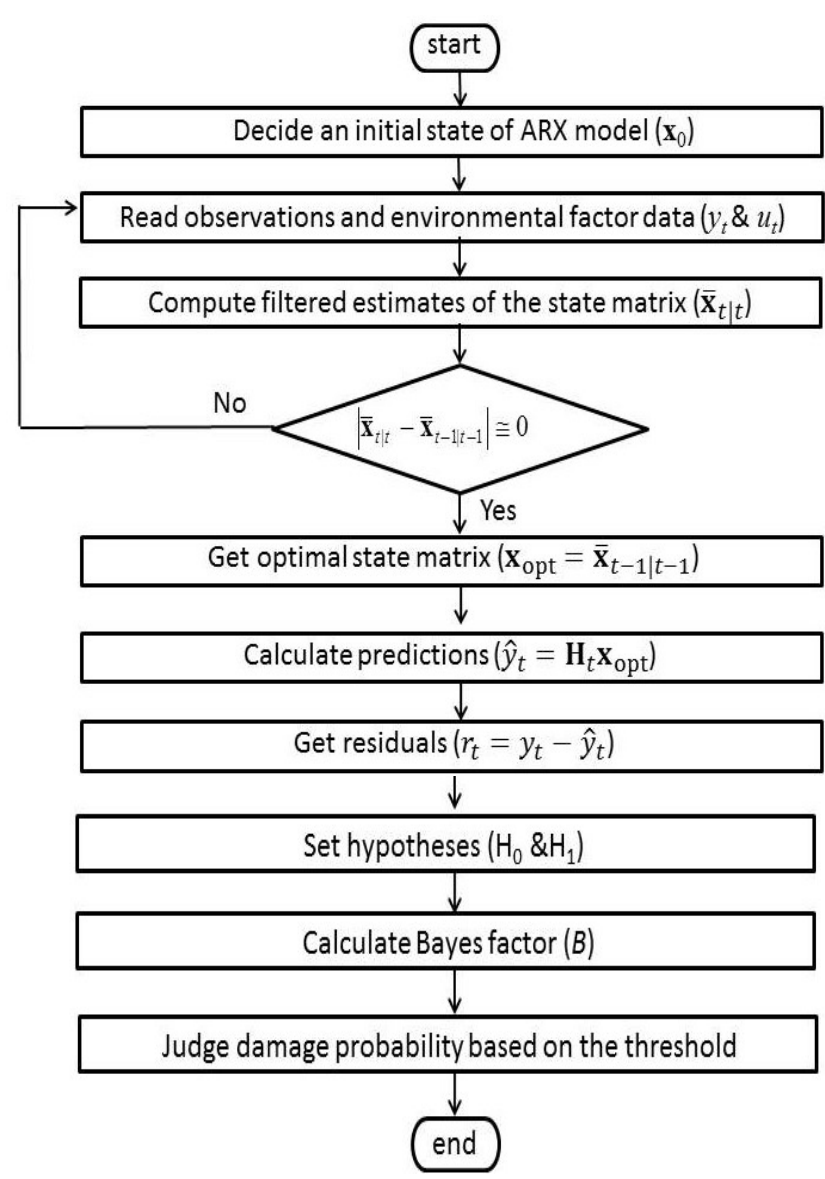

Figure. 1 Flowchart of the Bayesian approach for longterm bridge health monitoring.

We call the ratio of the marginal likelihoods as Bayes factor $(B)$, which is defined as the ratio of likelihood of the two scenarios 'damage' and 'healthy' as follows (e.g. Kass and Raftery 1995).

$B=\frac{p\left(\mathbf{D} \mid \mathrm{H}_{1}\right)}{p\left(\mathbf{D} \mid \mathrm{H}_{0}\right)}=\frac{\int_{0} p\left(\mathbf{D} \mid \theta_{1}, \mathrm{H}_{1}\right) p\left(\theta_{1} \mid \mathrm{H}_{1}\right) \mathrm{d} \theta_{1}}{\int_{0} p\left(\mathbf{D} \mid \theta_{0}, \mathrm{H}_{0}\right) p\left(\theta_{0} \mid \mathrm{H}_{0}\right) \mathrm{d} \theta_{0}}$

where $\theta_{0}$ and $\theta_{1}$ are parameters under $\mathrm{H}_{0}$ and $\mathrm{H}_{1}$.

If the Bayes factor is greater than 1, it implies that the data favor the hypothesis $\mathrm{H}_{1}$ and hence suggests that there is damage. If the Bayes factor is less than 1 , then there is no damage. According to Jeffreys (1998), a Bayes factor such that $1<B<3$ is 'barely worth mentioning', $3<B<10$ is 'substantial', $10<B<30$ is 'strong', $30<B<100$ is 'very strong', and $B>100$ is 'decisive'. In other words, $B<1$ is 'nothing (no damage)', $1<B<3$ is 'very small', $3<B<10$ is 'small', $10<B<30$ is 'strong', $30<B<100$ is 'very strong' and $B>100$ is 'decisive (damage)'. In practical applications, it might provide a threshold to decide an inspection, e.g. an emergency inspection if the Bayes factor gets more than 100 continuously, although more comprehensive studies are needed.

The flowchart of Bayesian approach for long-term bridge health monitoring is shown in Figure 1.

\subsubsection{Hypothesis test}

The $\mathrm{DI}_{o b}$ and $\mathrm{DI}_{p r}$ can be expressed as follows.

$$
\begin{aligned}
& D I_{o b}=D I_{t r}+\varepsilon_{o b} \\
& D I_{p r}=D I_{t r}+\varepsilon_{p r}
\end{aligned}
$$

where $\mathrm{DI}_{t r}$ indicates the true DI, $\varepsilon_{o b}$ and $\varepsilon_{p r}$ are normally distributed errors of $\mathrm{DI}_{o b}$ and $\mathrm{DI}_{p r}$ respectively. The residual can be expressed, then, as follows.

$r=D I_{o b}-D I_{p r}=\varepsilon_{o b}-\varepsilon_{p r}=\varepsilon$

where $\varepsilon \sim N\left(\mu, \sigma^{2}\right)$.

As an example of applying the Bayesian hypothesis test, this study assumes that the null hypothesis is defined by Equation 27 and the alternate hypothesis is defined by Equation 28.

$$
\begin{aligned}
& \mathrm{H}_{0}: \mu=\mu_{0}=0 \\
& \mathrm{H}_{1}: \mu=\mu_{1} \neq 0
\end{aligned}
$$

Also, the standard deviation was assumed to be constant as follows.

$$
\sigma=\sigma_{0}
$$

This study adopts the equation for $B$ proposed by Jiang and Mahadevan (2008) shown in Equation 30 in which the prior probability of the mean $\mu$ is assumed following the normal distribution with mean of $\rho$ and standard deviation of $\tau$.

$$
\begin{array}{r}
B=\frac{\int_{-\infty}^{\infty} p\left(\mathbf{D} \mid \mu, \mathrm{H}_{1}\right) p\left(\mu \mid \mathrm{H}_{1}\right) \mathrm{d} \mu}{\int_{-\infty}^{\infty} p\left(\mathbf{D} \mid \mu, \mathrm{H}_{0}\right) p\left(\mu \mid \mathrm{H}_{0}\right) \mathrm{d} \mu} \\
=\frac{\frac{1}{\sqrt{2 \pi \tau^{2}}} \sqrt{\frac{\pi}{A}} \exp \left(\frac{B^{2}}{4 A}+C\right)}{\exp \left(-\frac{n\left(\bar{r}^{2}+S^{2}\right)}{2 \sigma^{2}}\right)}
\end{array}
$$

where,

$$
\begin{aligned}
& A=\frac{n \tau^{2}+\sigma^{2}}{2 \sigma^{2} \tau^{2}} \\
& B=\frac{n \tau^{2} \bar{r}+\sigma^{2} \rho}{\sigma^{2} \tau^{2}} \\
& C=\frac{-n \tau^{2} S^{2}-n \tau^{2} \bar{r}^{2}-\sigma^{2} \rho^{2}}{2 \sigma^{2} \tau^{2}} \\
& \bar{r}=\frac{1}{n} \sum_{i=1}^{n} r_{i}
\end{aligned}
$$



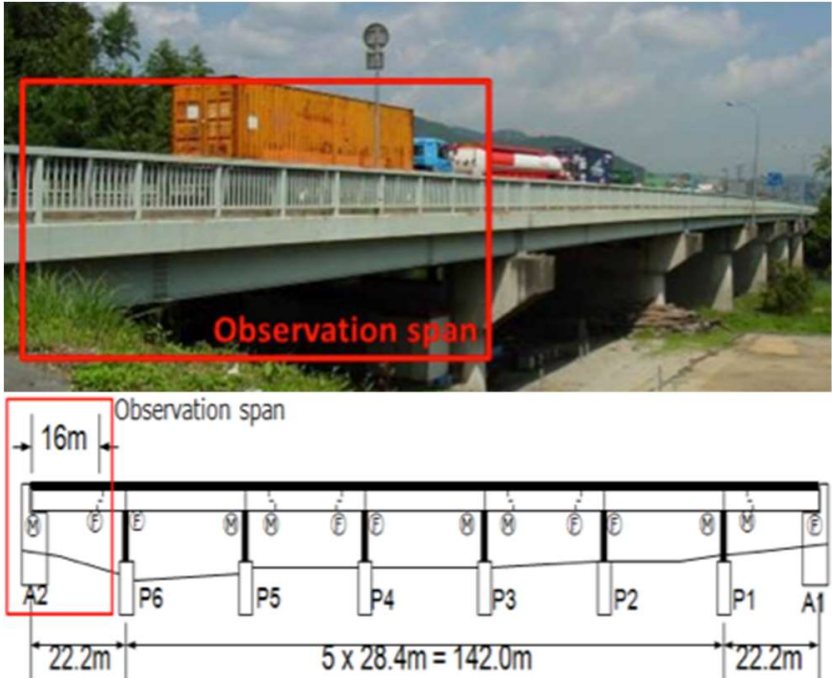

Figure 2. Observed bridge.

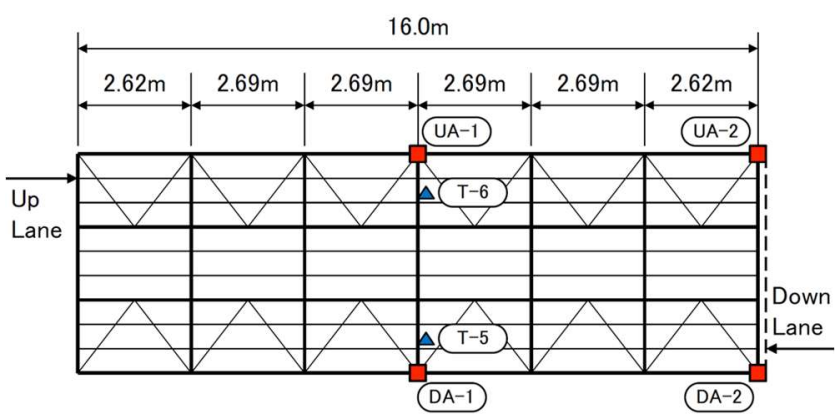

Figure 3. Sensor locations on the observation span.

Table 1. Properties of the observation bridge

\begin{tabular}{|c|c|c|}
\hline \multicolumn{2}{|c|}{ Construction year } & 1960 \\
\hline \multicolumn{2}{|c|}{ Bridge length (m) } & 186.4 \\
\hline \multirow[b]{2}{*}{ Span length (m) } & Hanging girder & 16.0 \\
\hline & $\begin{array}{l}\text { Anchorage } \\
\text { girder }\end{array}$ & $6.2+28.4+6.2$ \\
\hline \multicolumn{2}{|c|}{ Width (m) } & 8.0 \\
\hline
\end{tabular}

Table 2. Training and test data sets.

\begin{tabular}{ccc}
\hline $\begin{array}{c}\text { Name }(n: \\
\text { number of } \\
\text { data })\end{array}$ & Monitoring period & Monitored data \\
\hline $\begin{array}{c}\text { Data } 0 \\
(n=1512)\end{array}$ & $\begin{array}{c}\text { 5 August 2008- } \\
\text { 17 August 2009 }\end{array}$ & $\begin{array}{c}\text { Accelerations, } \\
\text { temperatures \& } \\
\text { vehicle weight }\end{array}$ \\
\hline Data 1 & 6 October 2009- & $\begin{array}{c}\text { Accelerations \& } \\
\text { temperatures }\end{array}$ \\
\hline$(n=700)$ & 29 March 2010 & $\begin{array}{c}\text { Accelerations \& } \\
\text { temperatures }\end{array}$ \\
\hline $\begin{array}{c}\text { Data } 2 \\
(n=1484)\end{array}$ & 2 July 2013- & 7 July 2014
\end{tabular}

Data 0: training data, Data1 \& Data 2: test data.

$S^{2}=\frac{1}{n} \sum_{i=1}^{n}\left(r_{i}-\bar{r}\right)^{2}$

$\mu \sim N\left(\rho, \tau^{2}\right)=\left(\frac{1}{2 \pi \tau^{2}}\right)^{\frac{1}{2}} \exp \left(-\frac{(\mu-\rho)^{2}}{2 \tau^{2}}\right)$

and $n$ denotes the number of the data and $r_{i}$ represents the $i$-th residual.

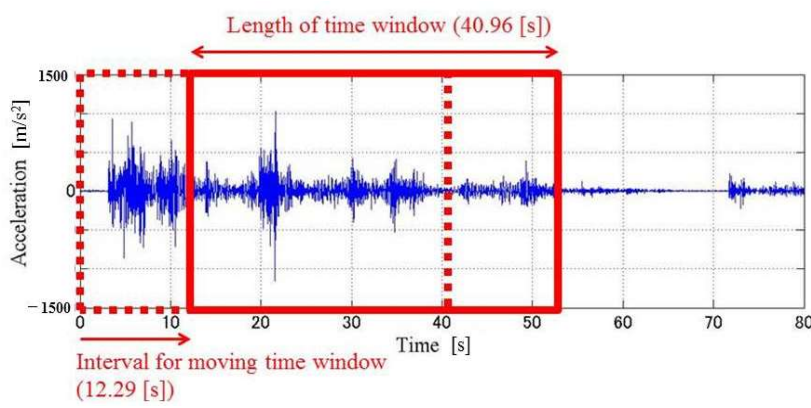

Figure 4. Time windows to identify the $\mathrm{DI}_{\mathrm{ob}}$ from acceleration measured at UA-1.
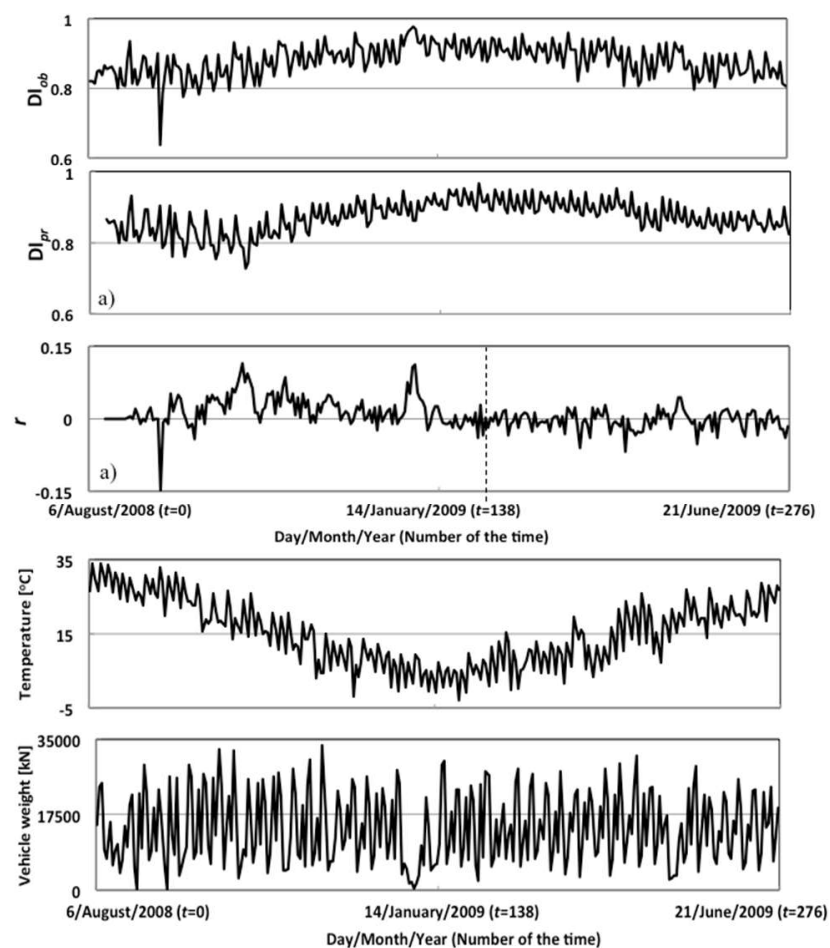

Figure 5. $\mathrm{DI}_{o b}, \mathrm{DI}_{p r}$ and residual of $\mathrm{DI}_{o b}$ and $\mathrm{DI}_{p r}$ identified at the sensor of UA-1, temperature measured at the sensor of T-6 and vehicle weight estimated by the BWIM system on up lane.

\section{LONG-TERM MONITORING ON AN IN- SERVICE PLATE-GERBER BRIDGE}

This study utilizes data monitored at the seven-span plate-Gerber bridge as shown in Figure 2, which is located on a busy national road in Japan.

The bridge properties are summarized in Table 1. The plan view with sensor locations on the observation span is shown in Figure 3. Therein, UA1, UA-2, DA-1 and DA-2 stand for accelerometers to measure acceleration responses of steel girders on up (UA) and down (DA) lanes. The sampling rate was $200 \mathrm{~Hz}$ for acceleration measurements. Thermometers were denoted by T-5 and T- 6 . Temperature was measured once every hour. A BWIM system (Moses 1979 and Heng et al. 2011) is installed in the bridge, and this study utilizes the vehicle's whole weight estimated by the BWIM system. 
This study focuses on the effects of time-varying temperature and vehicle weight measured at 1:00, 7:00, 13:00 and 19:00 on every Wednesday and Sunday for about one year (6th August 2008 to 21st June 2009) as a training data. It is noteworthy that all the data is taken from the healthy bridge since no damage and deterioration was reported during the monitoring period. Two other data sets are considered as test data. These one training data set and other two test data sets are shown in Table 2, where Data0 is the training data set and Datal and Data2 are the test data sets.

\section{APPLICATION AND DISCUSSION}

The proposed approach was applied to the long-term monitoring data and artificially made damage data.

\subsection{Regression analysis to consider environmental and operational changes}

This study identifies $100 \mathrm{DI}_{o b}$ 's from blocks of acceleration data obtaining by means of moving time windows as shown in Figure 4. This study adopted $40.96 \mathrm{~s}$ as the moving window following preliminary investigations considering different width of moving windows such as 20.48s, 40.96s and $81.92 \mathrm{~s}$ which showed similar trends with each other. For the overlap window, $70 \%$ overlap $(28.67 \mathrm{~s}$, in other words 12.29 s for un-overlap window length) was adopted in this study.

The $\mathrm{DI}_{o b}$ of Data 0 are shown in Figure 5 with predicted $\mathrm{DI}_{p r}$, residuals, temperature and vehicle weight. With Data 0, comparison was made between the linear regression and ARX considering only temperature. The AIC was also used as the index of regression accuracy since the smaller AIC is, the better the regression accuracy is. The results are shown in Figure 6. Therein the ARX model clearly showed better regression accuracy than that of the linear regression.

The AIC of four other cases considering different environmental and operational inputs shown in Table 3 were estimated and summarized in Figure 7, which implies that considering both temperature and vehicle weight resulted in better accuracy than consider only temperature. It is expected that considering both temperature and vehicle weight will have better accuracy than considering only vehicle weight although it was not demonstrated in this study.
Table 3. Different exogenous inputs.

\begin{tabular}{|c|c|c|c|}
\hline Name & $\begin{array}{l}\text { Regression } \\
\text { model }\end{array}$ & $\begin{array}{l}\text { Considered } \\
\text { factors }\end{array}$ & Utilized data \\
\hline ARX-T & ARX & Temperature & $\begin{array}{c}\text { Acceleration \& } \\
\text { temperature }\end{array}$ \\
\hline $\mathrm{AR}$ & $\mathrm{AR}$ & Not considered & Acceleration \\
\hline ARX-TW & ARX & $\begin{array}{l}\text { Temperature \& } \\
\text { vehicle weight }\end{array}$ & $\begin{array}{c}\text { Acceleration, } \\
\text { temperature } \\
\text { \& vehicle weight }\end{array}$ \\
\hline ARX-W & ARX & Vehicle weight & $\begin{array}{l}\text { Acceleration \& } \\
\text { vehicle weight }\end{array}$ \\
\hline
\end{tabular}

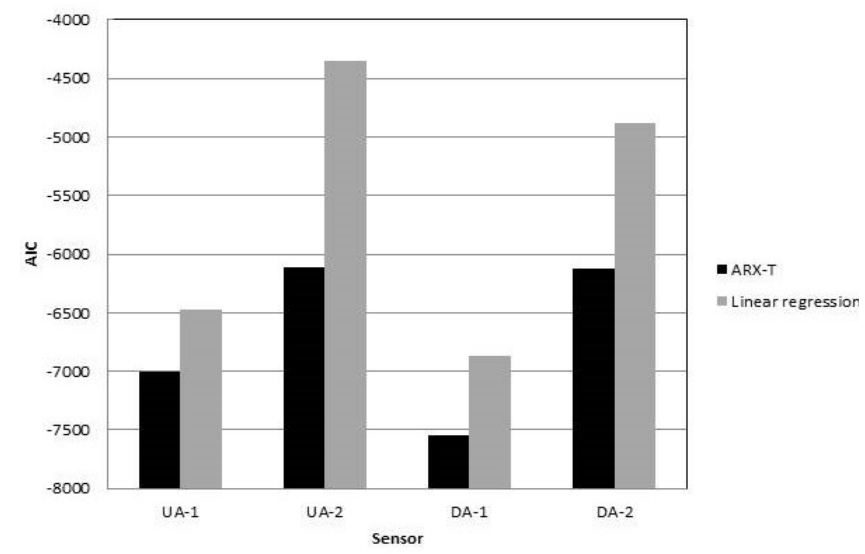

Figure 6. AIC utilizing ARX model and linear regression considering temperature at the sensors of UA-1, UA-2, DA1 and DA-2 in Data 0.

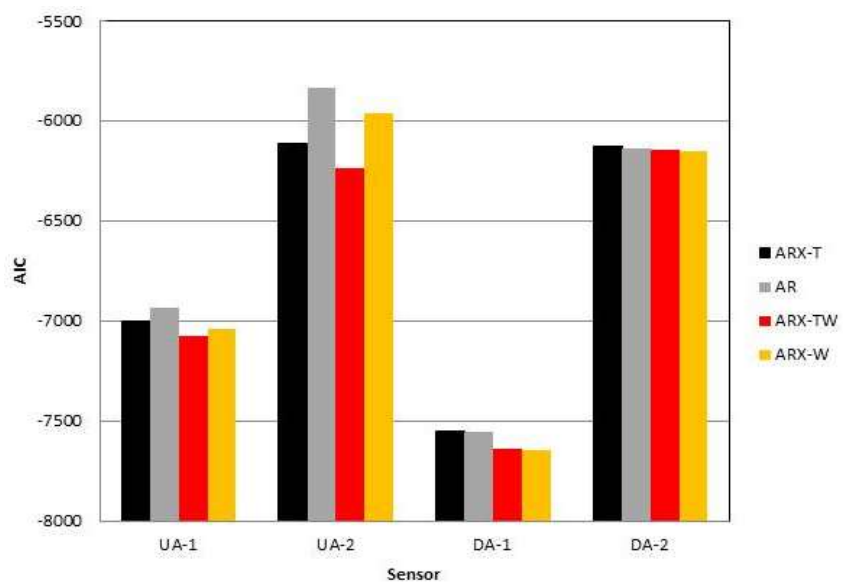

Figure 7. AIC utilizing ARX model in other cases at the sensors of UA-1, UA-2, DA-1 and DA-2 in Data 0.

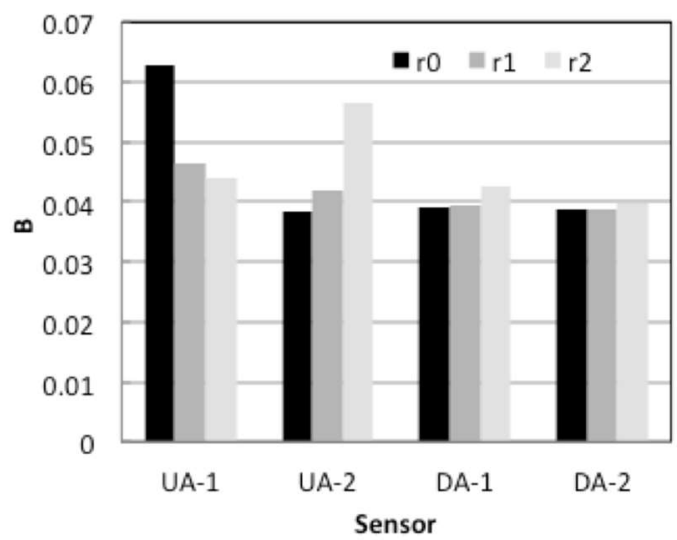

Figure 8. Bayes factors (B) at the sensors of UA-1, UA-2, DA-1 and DA-2 utilizing data from October to March in Data 0, Data 1 and Data 2. 


\subsection{Bayes factor for damage detection}

\subsubsection{With healthy data}

Data from October to March of Data 0, Data 1 and Data2 were utilized to calculate the Bayes factor (B). Only temperature was considered with the ARX model. The Bayes factors are summarized in Figure 8 , where $r 0, r 1$ and $r 2$ stand for the residuals of DI of Data 0 , Data 1 and Data 2 respectively.

In all cases, possibility of damage turned out to be very low. However, in all three other cases except UA-1, Bayes factor more or less shows increasing trend.

Those three sets of the monitored data at UA-1 and updated Bayes factors are shown in Figure 9, which also demonstrated that little possibility of damage. An interesting observation is that relatively larger changes of Bayes were observed at beginning of January of 2010 and 2014 because of the drastically reduced heavy traffics during new-year holidays. However after new-year holidays those changes were disappeared. It demonstrates that if increased Bayes factor will not decrease for a long time it indicates possibility of damage in the bridge.

\subsubsection{With artificially made damaged data}

Since data of a damaged bridge are not available, 300 random residuals were artificially generated by means of the Monte Carlo simulation and treated as damaged data. The residual were assumed as normally distributed with average of 0.01 (not 0 deliberately) and standard deviation of 0.02 . They were then connected with Data 0 as shown in Figure 10. These data were treated as continued data and the Bayes Factor was estimated and also plotted in Figure 10. It shows that the Bayes factor substantially increased in the area of damaged data, which implies possibility of the Bayes factor for damage detection.

\section{CONCLUSIONS}

This study investigated a way to consider changes in temperature and vehicle weight as environmental and operational factors for long-term vibrationbased BHM by applying the Bayesian approach to long-term monitoring data taken from an in-service plate-Gerber bridge. The Bayesian approach consists of three steps: Step 1 is to identify damage-sensitive features from coefficients of the AR model utilizing bridge accelerations; Step 2 is to consider environmental and operational changes by means of the Kalman filter; Step 3 is to make a decision based on the residuals utilizing the Bayesian hypothesis test.
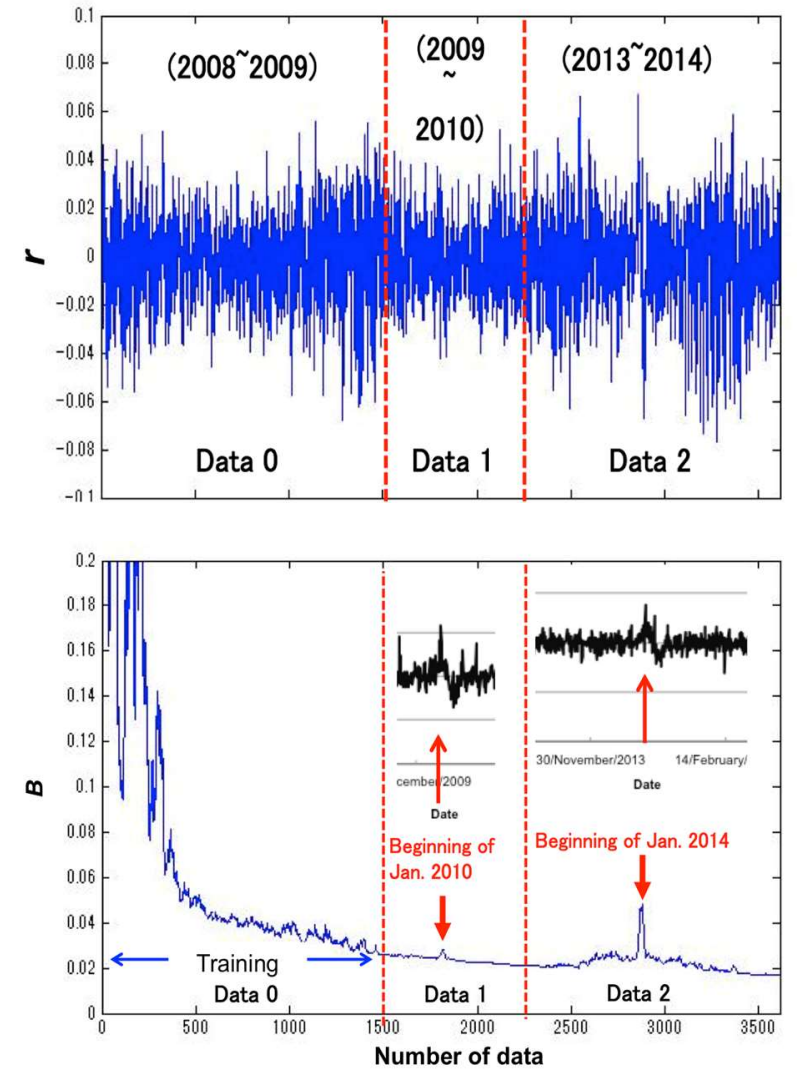

Figure 9. Three sets of acceleration data and updated Bayes factor w.r.t. time.

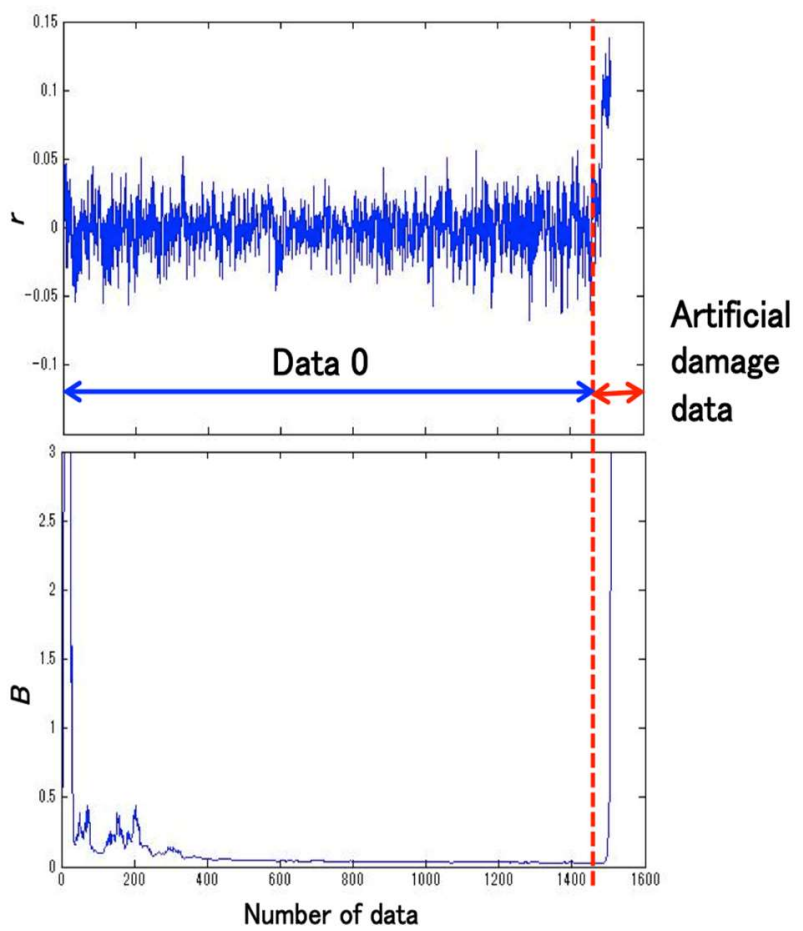

Figure 10. Three sets of acceleration data and updated Bayes factor w.r.t. time.

Observations through this study could be summarized as follows.

(1) The adoption of ARX model to consider environmental and operational changes led to better regression accuracy than linear regression.

(2) Observations demonstrated that possibility of damage is very low. 
(3) Gradual deterioration of the bridge might be progressing.

(4) Bayes factor might be a useful indicator for damage detection, but a definite conclusion still requires further investigations.

\section{REFERENCES}

Akaike, H. 1974. A new look at the statistical model identification. IEEE Transactions on Automatic Control, 19(6), 716-723.

Deraemaeker, A., Reynders, E., De Roeck, G. and Kullaa, J. 2007. Vibration-based structural health monitoring using output-only measurements under changing environment. Mech. Systems Signal Processing, 22(1):34-56.

Dilena, M. and Morassi, A. 2011. Dynamic testing of damaged bridge. Mech. Systems Signal Processing, 25:1485-1507.

Doebling, S.W., Farrar, C.R., Prime, M.B. and Shevitz, D.W. 1996. Damage identification and health monitoring of structural and mechanical systems from changes in their vibration characteristics: A literature review. Los Alamos National Laboratory Report, LA-3070-MS.

Cunha, A., Caetano, E., Magalhaes, F. and Moutinho, C. 2013. Recent perspectives in dynamic testing and monitoring of bridges. Struct. Control Health Monitoring, 20(6):853-877.

Heng, S., Oshima, Y. and Kawano, H. 2011. One Year Monitoring of Bridge Frequency and Traffic Load on a Road Bridge. Proc. of 24th KKCNN Symp. on Civil Eng.

Jeffreys, H. 1998. Theory of Probability. Oxford University Press Inc., New York, First published in the Oxford Classics series.

Jiang, X. and Mahadevan, S. 2008. Bayesian wavelet methodology for structural damage detection. Struct. Control Health Monitoring. 15:974-991.

Kass, R. and Raftery, A. 1995. Bayes factors. J. American Statistical Association. 90(430):773-795.

Kim, C.W., Sakakibara, T., Isemoto, R., Salpisoth, H., Oshima, Y. and Sugiura, K. 2011. One year vibration monitoring of a short span bridge under in-service environments. Proc. of 5th Int. Conf. on Structural Health Monitoring of Intelligent Infrastructure (SHMII-5), Cancun, Mexico (CDROM).

Kim, C.W., Kawatani, M. and Hao, J. 2012. Modal parameter identification of short span bridges under a moving vehicle by means of multivariate AR model. Struct. Infrastructure Eng. 8(5): 459-472.

Kim, C.W., Kitauchi, S., Sugiura, K. and Kawatani, M. 2013a. A year-long monitoring using in-service vibration data from a multi-span plate-Gerber bridge. Proc. of Life-Cycle and Sustainability of Civil Infrastructure Systems, Strauss, Frangopol and Bergmeister (Eds.):252-259.

Kim, C.W., Isemoto, R., Sugiura, K. and Kawatani, M. 2013 b. Structural fault detection of bridges based on linear system parameter and MTS method. J. JSCE. 1(1):32-43.

Kim, C.W., Morita,T., Oshima, Y. and Sugiura, K. 2015. A Bayesian approach for vibration-based long-term bridge monitoring to consider environmental and operational changes, Smart Structures and Systems, An Int J., 15(2): 395-408.

Kitagawa, G. and Gersch, W. 1984. A smoothness priors-state space modeling of time series with trend and seasonality. $J$. American Statistical Association, 79: 378-389.

Moses, F. 1979. Weigh-In-Motion System Using Instrumented Bridges. Transportation Eng. J., 105, TE3.

Nair, K.K., Kiremidjian, A.S. and Law, K.H. 2006. Time series-based damage detection and localization algorithm with application to the ASCE benchmark structure. Sound Vibration. 291:349-368.

Peeters, B. and De Roeck, G. 2001. One-year monitoring of the Z24-Bridge: environmental effects versus damage events. Earthquake Eng. Struct. Dynamics. 30:149-171.

Sankararaman, S. and Mahadevan, S. 2011. Bayesian methodology for diagnosis uncertainty quantification and health monitoring. Struct. Control Health Monitoring, 19:88-106.

Sohn, H., Worden, K. and Farrar, C.R. 2003. Statistical damage classification under changing environmental and operational conditions. Intelligent Material Systems Struct., 13:153-160. 